Check for updates

The BMJ

Cite this as: BMJ 2021;373:n1068 http://dx.doi.org/10.1136/bmi.n1068 Published: 23 April 2021

\section{Covid-19: Infections fell by $65 \%$ after first dose of AstraZeneca or Pfizer vaccine, data show}

\author{
Gareth lacobucci
}

Infections of SARS-CoV-2 fell by $65 \%$ after a first dose of the Oxford-AstraZeneca or Pfizer-BioNTech vaccines, preliminary results from a large UK surveillance study indicate.

Reductions increased to $70 \%$ after a second dose of the Pfizer vaccine, data from the UK Covid-19 Infection Survey show. Not enough people had yet received two doses of the AstraZeneca vaccine to assess this.

The survey, carried out by the University of Oxford in partnership with the Office for National Statistics and the Department of Health and Social Care for England, included data from 1.7 million self-reported swab test results taken from 370 ooo UK adults between 1 December 2020 and 3 April 2021.

The results, published in two preprint papers, ${ }^{12}$ show that two doses of the Pfizer vaccine offered levels of protection against covid-19 that were similar to levels from previous SARS-CoV-2 infection.

The benefits seem from the vaccines were similar in people over 75 and under 75 and in those with or without long term health conditions, the study found.

The researchers also found no evidence that the AstraZeneca and Pfizer vaccines differed in their ability to reduce infection rates $(\mathrm{P}>0.9)$, despite them leading to slightly different immune responses.

All participants showed at least some response to the vaccines, but the researchers noted that in the case of both vaccines a small percentage of people (around $5 \%$ ) had a low response and said that it would be essential to monitor their response to a second vaccine dose.

The study found that 21 days after a single dose of either the AstraZeneca or the Pfizer vaccine the rates of all new SARS-CoV-2 infections had fallen by $65 \%$ (95\% confidence interval 60\% to $70 \%$ ), symptomatic infections by $72 \%$ (69\% to $74 \%$ ), and asymptomatic infections by $57 \%$ (64\% to $47 \%)(\mathrm{P}<0.001$ for all).

Among people who had a second dose of the Pfizer vaccine, infections were $70 \%$ (62\% to $77 \%$; $\mathrm{P}<0.001)$ lower and symptomatic infections 90\% (82\% to 94\%; $\mathrm{P}<0.001)$ lower, similar to the effects in people who had previously been infected naturally ( $70 \%$ and $87 \%$ reductions, respectively).

The study found that both vaccines seemed to be highly effective against infections compatible with the Kent variant (B.1.1.7).

Koen Pouwels, senior researcher in Oxford University's Nuffield Department of Population Health and co-lead author of the study, said, "The protection from new infections gained from a single dose supports the decision to extend the time between first and second doses to 12 weeks to maximise initial vaccination coverage and reduce hospitalisations and deaths.

"However, the fact that we saw smaller reductions in asymptomatic infections than infections with symptoms highlights the potential for vaccinated individuals to get covid-19 again, and for limited ongoing transmission from vaccinated individuals, even if this is at a lower rate.”

\section{Antibody levels}

The second study compared how antibody concentrations changed after a single dose of either the AstraZeneca or Pfizer vaccine, or after two doses of the Pfizer vaccine (generally given 21-42 days apart), among 45965 people in the survey.

In those who had not previously had covid-19, antibody responses to a single dose of either vaccine were lower in older people, especially those aged over 60 years.

Two Pfizer doses achieved high antibody responses across all ages, particularly increasing seroconversion in older people, to levels similar to those achieved after prior infection followed by a single dose.

Antibody concentrations rose more slowly and to a lower level with a single dose of the AstraZeneca vaccine than with Pfizer's. However, they then dropped more quickly after a single Pfizer dose, reaching similar levels to those with a single dose of AstraZeneca, particularly among people at older ages.

But while the size of the immune response differed, no groups of individuals were found not to have responded at all to either vaccine.

Sarah Walker, professor of medical statistics and epidemiology at Oxford University and chief investigator for the UK Covid-19 Infection Survey, said, "We don't yet know exactly how much of an antibody response, and for how long, is needed to protect people against getting covid-19 in the long term-but over the next year information from the survey should help us to answer these questions."

Pritchard E, Matthews PC, Stoesser N, Eyre DW, Gethings O. Impact of vaccination on SARS-CoV-2 cases in the community: a population-based study using the UK's Covid-19 Infection Survey.

https:/www.ndm.ox.ac.uk/covid-19/covid-19-infection-survey/results/longer-articles/vaccine-effectiveness.

2 Weil, Stoesser N, Matthews PC, Studley R, Bell I. The impact of SARS-CoV-2 vaccines on antibody responses in the general population in the United Kingdom. https://www.ndm.ox.ac.uk/covid-19/covid-19-infection-survey/results/longer-articles/vaccine-effectiveness.

This article is made freely available for use in accordance with BMJ's website terms and conditions for the duration of the covid-19 pandemic or until otherwise determined by BMJ. You may use, download and print the article for any lawful, 
non-commercial purpose (including text and data mining) provided that all copyright notices and trade marks are retained. 